\title{
Análise da integração dos sistemas de gestão normalizados ISO 9001 e OHSAS 18001: Estudo de casos múltiplos
}

\section{Analysis of the integration of normalized management systems ISO 9001 and OHSAS 18001: Multiple case studies}

\author{
Gislaine Aparecida Vitoreli ${ }^{1}$ \\ Luiz Cesar Ribeiro Carpinetti ${ }^{1}$
}

\begin{abstract}
Resumo: Para atender às demandas do ambiente, as organizações implementam diversos sistemas de gestão. Com isso, dificuldades relacionadas ao seu gerenciamento paralelo podem surgir, o que leva as empresas a integrar estes sistemas em um único Sistema de Gestão Integrado (SGI). Dado este cenário, os pesquisadores buscam elaborar modelos que visam facilitar a implementação do SGI, a integração da documentação, além de investigar os benefícios e dificuldades dessa implementação. Entretanto, as pesquisas não mostram como a integração dos sistemas de gestão normalizados acontece nas organizações, assim uma investigação deste processo se torna interessante de forma a contribuir para a pesquisa sobre SGI. Com foco nos sistemas de gestão normalizados ISO 9001 e OHSAS 18001, o objetivo deste trabalho é analisar a integração desses sistemas de gestão sob três variáveis de pesquisa: o processo de implementação do SGI, a integração dos requisitos das normas e a estrutura de gestão do SGI. Para a condução desta pesquisa foi utilizado como método o estudo de caso. Como resultados, foram observadas diversas semelhanças no processo de implementação do SGI e na documentação desenvolvida pelas organizações para a sua implementação. Identificou-se ainda, que o tamanho da organização pode influenciar na estrutura de gestão estabelecida para o gerenciamento do SGI.
\end{abstract}

Palavras-chave: Sistema de gestão normalizado. Integração. OHSAS 18001. ISO 9001. Sistema de Gestão Integrado.

\begin{abstract}
In order to meet the demands of the environment, organizations usually implement a range of management systems. However, difficulties related to the parallel management of these systems may arise, and the companies are then driven to integrate their management systems into a single Integrated Management System (IMS). Given this scenario, researchers have designed models to ease the implementation of an IMS and the integration of documents and have investigated the benefits and difficulties of such an implementation. However, these studies have not shown how the integration of management systems takes place in organizations; therefore, the investigation of this process seems to be an interesting area to study contributing to IMS research. Focusing on the normalized management systems ISO 9001 and OHSAS 18001, this paper analyzes their integration by means of a case study based on three variables: IMS implementation process, integration of the IMS management norm requirements and structure. The results show similarities between the IMS implementation process and the documentation related to the IMS established by the organizations. It was also found that the size of the organization can affect the structure established to oversee the IMS management.
\end{abstract}

Keywords: Normalized management system. Integration. OHSAS 18001. ISO 9001. Integrated management system.

\section{Introdução}

A crescente necessidade das organizações em estabelecer um bom relacionamento com diversos stakeholders tem levado à adoção, cada vez mais frequente, de diferentes sistemas de gestão, cada um cobrindo requisitos mínimos para o atendimento de determinado objetivo (ZUTSHI; SOHAL, 2005). Isto pode ser visualizado por meio do aumento no número de certificações, sendo que, mundialmente, o número de certificados ISO 9001 emitidos passou de 497.919 em Dezembro de 2003 para 951.486 em Dezembro de 2007. Já o número de certificados ISO 14001 passou de 111.162 em Dezembro de 2005 para 154.572 em Dezembro de 2007 (INTERNATIONAL..., 2009).

\footnotetext{
${ }^{1}$ Gestão da Qualidade e Mudança - GQM, Programa de Pós-graduação em Engenharia de Produção, Departamento de Engenharia de Produção, Escola de Engenharia de São Carlos - EESC, Universidade de São Paulo - USP, Av. Trabalhador São-Carlense, 400, Centro, CEP 13566-590, São Carlos, SP, Brasil, e-mail: gislaineavitoreli@gmail.com.br; carpinet@ sc.usp.br
} 
Com o aumento na adoção de diferentes sistemas de gestão pelas organizações, surgem dificuldades relacionadas ao gerenciamento paralelo destes sistemas, assim a integração é vista como uma forma de gerar maior eficiência em diversos aspectos, amenizando estas dificuldades (KARAPETROVIC, 2003; JONKER; KARAPETROVIC, 2004; ZUTSHI; SOHAL, 2005; WILKINSON; DALE, 1999; POJASEK, 2006). Salomone (2008) ressalta que, além da necessidade de integração devido às dificuldades referentes ao gerenciamento, isto também ocorre devido às vantagens que podem ser obtidas, como a redução de diversos custos (e.g. aqueles relacionados à auditoria externa, que passa a ser realizada de maneira integrada).

Apesar das vantagens que as organizações podem alcançar, diversos problemas tendem a surgir durante o processo de integração. Esses problemas, muitas vezes, são influenciados por fatores como as diferentes abordagens utilizadas pelas normas de sistemas de gestão (e.g. abordagem de processos, ciclo PDCA - Plan/Do/Check/Act), os requisitos específicos de cada função (e.g. qualidade, meio ambiente, segurança e saúde ocupacional) e, finalmente, pela falta de pessoas que possuam conhecimento nas várias funções abordadas pelos sistemas de gestão (KARAPETROVIC, 2003; SALOMONE, 2008).

Devido às dificuldades citadas anteriormente, pesquisas buscam medidas para facilitar esse processo, como a elaboração de modelos para auxiliar na integração das normas e também na própria implementação dos sistemas de gestão integrados (JONKER; KARAPETROVIC, 2004; ZENG; SHI; LOU, 2007; KARAPETROVIC, 2003). Já os estudos empíricos sobre Sistemas de Gestão Integrados (SGIs) tratam de questões como as vantagens e benefícios gerados com a integração (ZUTSHI; SOHAL, 2005), os fatores que afetam a integração dos sistemas de gestão, os principais problemas no gerenciamento de sistemas de gestão paralelos (ZENG; SHI; LOU, 2007), o potencial de integração das normas (SALOMONE, 2008) e a extensão com que as empresas integram seus sistemas de gestão (BERNARDO et al., 2009).

Desta forma, a bibliografia da área trata de diversas soluções para a questão da integração de sistemas de gestão, propõe passos para a sua implementação e estudos empíricos apresentam questões mais amplas sobre o tema, mas sem apresentar de maneira detalhada a forma como acontece a integração de sistemas de gestão normalizados nas organizações. Assim, identificou-se que uma investigação detalhada de como esse processo ocorre se mostra interessante de modo a contribuir para o conhecimento na área.

Para a realização desta investigação, é necessário o estabelecimento de um escopo, ou seja, é necessário identificar um número limitado de normas de sistemas de gestão para que a análise seja realizada.
Essa definição é necessária uma vez que diversas organizações podem adotar um conjunto distinto de normas de sistemas de gestão (JONKER; KARAPETROVIC, 2004; KARAPETROVIC, 2003), e uma comparação entre os processos de integração é facilitada pela adoção de uma amostra homogênea (POUPART et al., 2008).

Assim, foi determinado como escopo da pesquisa organizações que possuam, ao menos, as certificações ISO 9001 e OHSAS 18001 e que integrem, ao menos, estes sistemas de gestão. Quanto à integração da ISO 9001 e a OHSAS 18001, os trabalhos encontrados analisam a compatibilidade entre as normas para a integração utilizando como método de pesquisa o survey (PHENG; PONG, 2003), além de identificar, entre empresas certificadas ISO 9001, OHSAS $18001 \mathrm{e}$ ISO 14001 (PHENG; KWANG, 2005), as dificuldades, benefícios e custos da integração. Conforme pode ser observado, a bibliografia não apresenta como acontece o processo de integração dos sistemas de gestão ISO 9001 e OHSAS 18001, fazendo com que exista uma motivação teórica para a escolha desses sistemas para a realização da investigação.

Assim, este trabalho tem com objetivo descrever e analisar o processo de integração dos sistemas de gestão normalizados ISO 9001 e OHSAS 18001 em empresas certificadas nessas normas. Para a realização desta análise, foram estabelecidas três variáveis de pesquisa que se referem aos aspectos considerados relevantes quando se fala na integração de sistemas de gestão. São elas: o processo de implementação do SGI, a integração dos requisitos das normas e a estrutura de gestão do SGI.

Por meio dessa análise, espera-se contribuir para as áreas acadêmica, descrevendo e analisando o processo de integração dos sistemas de gestão normalizados ISO 9001 e OHSAS 18001, e gerencial, apresentando como ocorre o processo de integração desses sistemas em empresas certificadas nessas normas, o que pode servir como referência para empresas que pretendam integrar os seus sistemas de gestão.

\section{Sistemas de gestão}

Para compreender o conceito de sistema de gestão, é importante entender primeiramente o conceito de sistema. Segundo Churchman (1972), um sistema é um conjunto de partes coordenadas para realizar um conjunto de finalidades, definição similar às apresentadas por outros autores (INTERNATIONAL..., 2010; JONKER; KARAPETROVIC, 2004). Apesar das várias definições existentes, de maneira geral, os sistemas são vistos como um conjunto de partes coordenadas que interagem de forma a atingir determinado(s) objetivo(s) (RIBEIRO NETO; TAVARES; HOFFMANN, 2008).

Os sistemas de gestão são um tipo de sistema formado por partes (requisitos), que se relacionam 
entre si, de forma a atender a um determinado objetivo, o qual está relacionado à função do sistema de gestão. Por exemplo, um sistema de gestão da qualidade tem como um de seus objetivos fornecer produtos que atendam aos requisitos dos clientes. Já um sistema de gestão de segurança e saúde ocupacional pode ter como objetivo controlar os riscos de Segurança e Saúde Ocupacional (SSO) e melhorar continuamente as condições de SSO nas organizações (RIBEIRO NETO; TAVARES; HOFFMANN, 2008).

Os exemplos de objetivos de sistemas de gestão citados anteriormente referem-se aos sistemas de gestão normalizados ISO 9001 e OHSAS 18001. Sistemas de gestão normalizados são aqueles baseados em normas, de caráter nacional ou internacional, elaboradas devido à necessidade das organizações em atender à demanda de diversos grupos de interesse (ZUTSHI; SOHAL, 2005). Assim, a normalização pode ser entendida como uma prescrição para a utilização de práticas relacionadas à determinada área de gestão (e.g. qualidade, segurança e saúde ocupacional, responsabilidade social), visando o atendimento da demanda dos diversos grupos de interesse que cercam a organização (ASSOCIAÇÃO..., 2009).

Dado o conceito de sistemas de gestão e apresentada a importância da normalização, a próxima seção apresenta algumas características dos sistemas de gestão normalizados ISO 9001 e OHSAS 18001, os quais são o foco deste trabalho.

\subsection{Os sistemas de gestão da qualidade (ISO 9001) e de segurança e saúde ocupacional (OHSAS 18001)}

A ISO 9001 faz parte da série de normas ISO 9000 e é caracterizada por ser uma norma genérica que pode ser utilizada por qualquer organização para o estabelecimento de um sistema de gestão da qualidade, podendo ser certificada por um organismo externo (INTERNATIONAL..., 2009). Seus requisitos são baseados nos princípios de gestão da qualidade total: foco no cliente, liderança, envolvimento das pessoas, abordagem de processo, abordagem sistêmica para a gestão, melhoria contínua, tomada de decisão baseada em fatos e benefícios mútuos nas relações com os fornecedores (TRICKER, 2007).

Os requisitos da norma ISO 9001 estão divididos em cinco seções: sistema de gestão da qualidade; responsabilidade da direção; gestão de recursos; realização do produto e medição; análise e melhoria (ASSOCIAÇÃ̃O..., 2008). As ligações entre estas seções podem ser descritas da seguinte forma: a alta direção tem como responsabilidade prover os recursos necessários (infraestrutura, capacitação de pessoal, ambiente de trabalho adequado, dentre outros) para o processo de realização do produto, que deve operar de maneira a atender aos requisitos estabelecidos pelos clientes. Depois de sua fabricação, o produto é entregue aos clientes e a organização deve monitorar a sua satisfação por meio de indicadores, dados que alimentarão o processo de medição, análise e melhoria contínua do sistema de gestão da qualidade.

A OHSAS 18001 apresenta requisitos para o gerenciamento da segurança e saúde ocupacional na organização e foi desenvolvida por uma seleção dos principais organismos de comércio, normas internacionais e organismos de certificação para preencher uma lacuna da falta de uma norma internacional de segurança e saúde ocupacional (BRITISH..., 2007). A norma OHSAS 18001 foi desenvolvida com base no ciclo PDCA (Plan-Do-Check-Act ou Planejar, Fazer, Verificar e Agir), sendo que seus requisitos podem ser relacionados a cada uma das etapas deste ciclo (KAUSEK, 2007).

Os requisitos de planejamento (Etapa Planejar) incluem o desenvolvimento de uma política de segurança e saúde ocupacional, identificação dos perigos e avaliação dos riscos do ambiente de trabalho e o planejamento de melhoria por meio de objetivos e metas de segurança e saúde. Já os requisitos relacionados à etapa "fazer" do ciclo incluem a implementação dos controles e medidas preventivas identificadas na primeira fase e o treinamento dos funcionários para que esses desenvolvam suas tarefas com segurança e estejam cientes da importância do sistema de gestão de segurança e saúde na organização (KAUSEK, 2007).

Por meio das ações corretivas e preventivas, etapa "verificar" do ciclo PDCA, a organização monitora os controles estabelecidos e o desempenho de seu Sistema de Gestão de Segurança e Saúde Ocupacional (SGSSO). Por fim, a etapa "agir" do ciclo é representada pelos requisitos de análise crítica da direção, devendo gerar ações para melhoria do SGSSO. Apresentadas algumas características das normas ISO 9001 e OHSAS 18001, o próximo tópico trata da questão da integração de sistemas de gestão.

\subsection{Sistemas de Gestão Integrados}

As organizações são consideradas sistemas abertos e, como tal, estão sujeitas às demandas do ambiente, o que gera a necessidade de sua constante adaptação (DESSLER, 1976). Uma das exigências do atual ambiente organizacional refere-se ao estabelecimento de um bom relacionamento com vários stakeholders, o que tem levado as organizações a adotarem diferentes sistemas de gestão (ZUTSHI; SOHAL, 2005). Devido às dificuldades de gerenciamento paralelo destes sistemas, a sua integração em um único Sistema de Gestão Integrado tem sido vista como uma forma de gerar diversos benefícios, como redução de custos e a melhoria no gerenciamento (KARAPETROVIC, 2003; JONKER; KARAPETROVIC, 2004; ZUTSHI; 
SOHAL, 2005; WILKINSON; DALE, 1999; POJASEK, 2006).

Os Sistemas de Gestão Integrados podem ser definidos como um conjunto de processos interrelacionados que dividem um conjunto de recursos humanos, financeiros, materiais, além de uma infraestrutura e informações, de forma a atingir um conjunto de objetivos relacionados à satisfação dos stakeholders (KARAPETROVIC; WILLBORN, 1998).

Devido à crescente importância atribuída à integração dos sistemas de gestão, a literatura apresenta diversos trabalhos sobre o tema, tanto teóricos, quanto empíricos. Os trabalhos empíricos buscam analisar a compatibilidade entre sistemas de gestão normalizados (PHENG; PONG, 2003), apresentam experiências de empresas com a implementação do SGI (FRESNER; ENGELHARDT, 2004; ZUTSHI; SOHAL, 2005), investigam o potencial para integração das normas, partindo da análise de aspectos comuns entre as empresas em termos de motivações, obstáculos e pressão externa quando da implementação de cada sistema de gestão (SALOMONE, 2008) e estudam em que extensão as empresas integram os seus sistemas de gestão (BERNARDO et al., 2009). Outros trabalhos estudam a integração de requisitos específicos das normas, como é o caso do trabalho de Bernardo et al. (2010), que busca entender como ocorre a integração das auditorias internas e externas nas organizações. Mais recentemente, Bernardo et al. (2012) analisam empiricamente a influência das dificuldades que surgem durante a integração e o nível de integração atingido pelas organizações.

Quanto aos trabalhos empíricos citados, é importante ressaltar os resultados comuns encontrados pelos autores. Como benefícios da integração dos sistemas de gestão, Zutshi e Sohal (2005) e Salomone (2008) destacam a unificação dos treinamentos, melhor utilização de recursos financeiros e unificação das auditorias, tanto internas como externas. A integração dos requisitos dos sistemas de gestão é tratada nos trabalhos de Salomone (2008) e Bernardo et al. (2009), em que o primeiro destaca os requisitos mais comumente submetidos à integração e o segundo trata do grau de integração dos requisitos. Analisando os resultados desses trabalhos, é possível identificar aqueles requisitos dos sistemas de gestão que as empresas integram mais e em maior grau. São eles: o controle de documentos, controle de registros, política, objetivos e metas, manual, auditoria interna, análise crítica da direção e comunicação interna.

Quanto ao trabalho de Bernardo et al. (2012), os resultados mostram que as dificuldades encontradas durante o processo de integração das normas afetam o nível de integração alcançado pelas empresas quando estas integram três sistemas de gestão. Já quando a integração é de dois sistemas de gestão não foi encontrada uma relação significativa entre as dificuldades encontradas e o nível de integração alcançado. As principais dificuldades identificadas pelos autores no processo de integração foram agrupadas em dois clusters: as internas (falta de recursos humanos, falta de colaboração entre os departamentos, falta de auditores especializados, falta de tempo para a integração e falta de motivação dos funcionários) e aquelas relacionadas com as normas (diferentes modelos de cada norma e diferenças em elementos comuns entre as normas). Segundo os autores, identificou-se que as dificuldades relacionadas com as normas são um pouco maiores do que as internas, mostrando que a questão das diferenças encontradas entre as normas afetam mais o nível de integração dos sistemas de gestão do que aquelas relacionadas a fatores internos.

Já os trabalhos teóricos sobre SGI abordam aspectos como a elaboração de modelos para auxiliar na integração das normas, as etapas para a implementação de um SGI e os níveis de integração (JONKER; KARAPETROVIC, 2004; ZENG; SHI; LOU, 2007; KARAPETROVIC, 2003; POJASEK, 2006; ØRGENSEN; REMMEN; MELLADO, 2006; SAMPAIO; SARAIVA; DOMINGUES, 2012). O trabalho de Jonker e Karapetrovic (2004) apresenta um modelo interessante para a integração dos requisitos das normas, baseado na abordagem de sistemas, que oferece a base para o agrupamento de diferentes sistemas de gestão em um sistema único. Por meio deste modelo, as organizações podem integrar os requisitos comuns entre as normas e formar o que os autores chamam de subsistemas específicos, os quais abrigam os requisitos específicos de cada norma.

Sobre o processo de implementação do SGI, Karapetrovic (2003) apresenta um fluxograma com várias etapas nas quais, inicialmente, a alta direção decide onde a integração é desejável e viável e escolhe um modelo para a integração. O próximo passo consiste na definição das funções (qualidade, meio ambiente, SSO) a serem incluídas no SGI e na escolha das normas a serem utilizadas. Realizadas as escolhas, os requisitos das normas devem ser integrados e, posteriormente, devem ser analisadas as lacunas entre os sistemas de gestão, ou seja, deve-se identificar pontos específicos que são abordados apenas por determinada norma. $\mathrm{O}$ alinhamento e a integração da documentação, dos objetivos específicos das funções e dos recursos e processos dos sistemas de gestão, são o próximo passo. O último passo refere-se à melhoria do sistema de gestão integrado, o que pode ser feito de maneira quantitativa ou qualitativa.

Por fim, o trabalho de Sampaio, Saraiva e Domingues (2012) propõe quatro níveis de integração dos sistemas de gestão: integração da documentação (nível 1), integração das ferramentas de gerenciamento (nível 2), políticas e objetivos comuns (nível 3) e estrutura organizacional comum (nível 4). Os 
autores ainda fazem uma série de recomendações para aquelas empresas que procuram evoluir na integração dos seus sistemas de gestão para que esta seja realizada de maneira eficiente e eficaz. Dentre estas recomendações destacam-se: adotar um sistema de gestão integrado flexível (ou seja, que permita a realização de mudanças necessárias à sua adaptação, por exemplo, a inclusão de novos sistemas de gestão), assegurar o comprometimento da alta direção, implementar atividades de treinamento integradas, realizar auditorias internas integradas e assegurar que existam recursos disponíveis para a manutenção do sistema de gestão integrado.

Ainda que a literatura apresente modelos para a integração de sistemas de gestão, não é possível afirmar que existe uma maneira ideal para o gerenciamento e estabelecimento de SGIs nas organizações, o que é coerente com o modelo da teoria da contingência (LAWRENCE; LORSCH, 1973; KAST; ROSENZWEIG, 1979). Assim, a forma como a integração dos sistemas de gestão acontece nas organizações, e quais sistemas de gestão são integrados, pode variar de empresa para empresa, pois cada uma delas está inserida em um determinado ambiente (LAWRENCE; LORSCH, 1973), em que os mais diferentes aspectos, como as exigências dos stakeholders, podem se modificar. Isto pode ser exemplificado por meio da exigência de certificações específicas por determinados mercados, como é o caso da certificação British Retail Consortium, um referencial de qualidade desenvolvido por grandes grupos comerciais britânicos, sendo que, todas as organizações que pretendem fornecer para estes grupos devem cumprir com os requisitos estabelecidos neste referencial (BRITISH..., 2010). Outras organizações podem optar por não integrar todos os seus sistemas de gestão em um único sistema, o que pode ocorrer devido à criticidade de determinado sistema de gestão para os negócios da empresa (KARAPETROVIC, 2003; SALOMONE, 2008). Outro aspecto importante relacionado à integração de sistemas de gestão normalizados, mas que não é amplamente discutido na literatura, é o desenvolvimento de uma estrutura para o gerenciamento do SGI. O trabalho de Zeng, Shi e Lou (2007) cita apenas a importância da existência de uma sinergia no gerenciamento do SGI, já o trabalho de Zutshi e Sohal (2005) ressalta a importância da existência de um responsável pelo SGI entre a média e a alta gerência. A adoção de vários sistemas de gestão requer que as empresas adaptem a sua estrutura de gerenciamento, de forma a alcançar maior eficiência na gestão destes sistemas. Para isso, deve existir uma coordenação entre os departamentos envolvidos no Sistema de Gestão Integrado. A questão da integração entre os departamentos das organizações foi amplamente discutida no trabalho de Lawrence e Lorsch (1973), que abordam os aspectos de diferenciação e integração administrativas nas organizações. Em seu trabalho, os autores identificaram a existência de unidades integradoras, as quais dizem respeito a estruturas formais ou informais estabelecidas na organização e que têm como objetivo integrar as atividades dos departamentos da empresa.

Com a realização da pesquisa, os autores concluíram que as organizações se diferenciam em partes, e o funcionamento destas partes separadas tem de ser integrado para que o sistema inteiro seja viável. Isso deve acontecer conforme requerido pelo ambiente externo e meios eficazes para a resolução de conflitos entre as partes devem ser utilizados. Em linhas gerais, ao invés de propor uma maneira única para que as empresas possam se organizar em quaisquer condições, os autores argumentam que isto deve ser feito de acordo com as suas características, e as características do ambiente em que atua, o que a leva a um desempenho eficaz (LAWRENCE; LORSCH, 1973).

Analisando o trabalho de Lawrence e Lorsch (1973) e observando a importância da existência de uma coordenação entre as áreas que compõem o SGI, acredita-se que a criação de unidades integradoras entre estas áreas seja importante para que ocorra melhor gerenciamento do SGI. É importante destacar que esta coordenação pode ocorrer de maneira diferente em cada organização, o que pode depender de fatores contingenciais não só relacionados ao ambiente industrial (LAWRENCE; LORSCH, 1973), mas também aos padrões adotados pelas organizações, que podem estar relacionados a questões como o processo de tomada de decisões, ao estabelecimento de padrões para comunicação ou comportamento e com o tamanho da organização (DONALDSON, 2007; BERTERO, 2007).

\section{Método de pesquisa}

Em relação à abordagem, esta pesquisa é classificada como qualitativa, uma vez que o seu foco é nos processos do objeto de estudo (MIGUEL, 2007) que, neste caso, refere-se ao processo de integração dos sistemas de gestão ISO 9001 e OHSAS 18001. Quanto aos objetivos, esta pesquisa pode ser classificada como descritiva exploratória. Segundo Miguel (2007), estes tipos de pesquisa (descritiva e exploratória) não são excludentes, sendo que se pode classificar uma pesquisa como exploratória, e, se não existe relação causal entre as suas variáveis, a pesquisa é também descritiva. Este é o caso deste trabalho, uma vez que as variáveis foram estabelecidas de forma que o SGI das unidades de análise pudesse ser descrito com foco em questões importantes sobre a integração identificadas na literatura e, posteriormente, analisado.

O estudo de caso foi adotado como método para o desenvolvimento da pesquisa, dado que ele é utilizado quando o propósito da pesquisa é descrever 
uma situação ou entender como e porque os eventos ocorrem (YIN, 2005). Optou-se por realizar um estudo de casos múltiplos (formado por 4 empresas), que possui como vantagem, em relação à adoção do estudo de caso único, o maior grau de generalização dos resultados (YIN, 2005).

Para a seleção dos estudos de caso, foi utilizada uma amostra intencional, ou seja, as empresas foram escolhidas devido à sua relevância para o assunto tratado nesta pesquisa. Segundo Yin (2005), o princípio da intencionalidade é adequado no contexto da pesquisa social com ênfase nos aspectos qualitativos, em que todas as unidades não são consideradas como equivalentes, ou de mesma relevância. Uma vez que este trabalho é sobre a integração dos sistemas de gestão normalizados ISO 9001 e OHSAS 18001, optou-se por estudar organizações que sejam certificadas, ao menos, nestas duas normas, as quais devem estar implementadas de maneira integrada.

Para identificar estas organizações, foram consultados a base de dados da Associação Brasileira de Normas Técnicas, que informa as empresas certificadas ISO 9001 no Brasil, e o Anuário Brasileiro de Proteção, revista especializada na área de segurança ocupacional, que elabora anualmente uma lista das empresas certificadas pela norma OHSAS 18001, utilizando como fonte para a elaboração desta lista as próprias empresas certificadas e organismos de certificação.

Para a coleta dos dados, foi utilizado um questionário semiestruturado, recomendado em casos em que o interesse do pesquisador é "desvendar o desenrolar dos eventos que culminam nos resultados" (MARTINS, 2010). O questionário foi organizado em quatro seções, as quais são apresentadas no Quadro 1.
Para a verificação da qualidade dos dados obtidos com a aplicação do questionário, foi realizado um teste-piloto, o qual também possibilita verificar se estes dados atendem aos objetivos de pesquisa (MIGUEL, 2010). Como resultados da realização do estudo de caso piloto, várias melhorias foram realizadas no questionário, dentre elas: reformulação de questões (com o objetivo de obter as informações desejadas e facilitar a compreensão das questões pelos entrevistados), acréscimos de questões (de forma a compreender melhor aspectos referentes à estrutura de gestão do SGI e obter outras informações sobre a percepção dos entrevistados quanto à implementação do SGI) e reorganização das questões no questionário (que foram divididas conforme as variáveis da pesquisa).

As fontes de evidência utilizadas para a realização desta pesquisa foram entrevistas, documentos e observações diretas. Os dados provenientes das entrevistas foram registrados por meio de um gravador, que, apesar de apresentar desvantagens, como a possibilidade de inibir o entrevistado devido ao seu uso, é um meio recomendado para o registro dos dados durante a condução de pesquisas por permitir a coleta de informações mais precisas (MIGUEL, 2007). No total, as entrevistas tiveram duração de 6 horas e 24 minutos, 5 horas e 16 minutos, 6 horas e 4 horas e 30 minutos, respectivamente, para as Unidades de Análise 1, 2, 3 e 4 . As entrevistas foram realizadas com mais de uma pessoa em todas as unidades de análise. Para a escolha dos entrevistados, observou-se a função desempenhada por eles, sendo que, a cada um deles, foram direcionas as questões relativas à sua função. Em todas as organizações, o responsável pelo SGI foi entrevistado.

Quadro 1. Características do questionário semiestruturado utilizado para a realização da pesquisa.

\begin{tabular}{|c|c|c|}
\hline Seção & Descrição & Referências \\
\hline $\begin{array}{l}\text { Seção 1. Dados do } \\
\text { entrevistado e perfil } \\
\text { da empresa }\end{array}$ & $\begin{array}{l}\text { Função, tempo no cargo e tempo na empresa do } \\
\text { entrevistado. Informações gerais sobre a empresa (produtos, } \\
\text { clientes, certificações, mercado de atuação e nacionalidade). }\end{array}$ & - \\
\hline $\begin{array}{l}\text { Seção 2. Integração } \\
\text { das normas ISO } 9001 \\
\text { e OHSAS } 18001\end{array}$ & $\begin{array}{l}\text { Seção subdividida em três itens: processo de implementação } \\
\text { do SGI, integração dos requisitos das normas e estrututra de } \\
\text { gestão do SGI. }\end{array}$ & \multirow{4}{*}{$\begin{array}{l}\text { Baseado em literatura da } \\
\text { área (ZUTSHI; SOHAL, } \\
\text { 2005; SALOMONE, } \\
\text { 2008; POJASEK, } \\
\text { 2006; ØRGENSEN; } \\
\text { REMMEN; } \\
\text { MELLADO, 2006; } \\
\text { PHENG; PONG, 2003; } \\
\text { KARAPETROVIC, } \\
\text { 2003) e na tabela de } \\
\text { correspondências de } \\
\text { requisitos apresentada } \\
\text { pela norma OHSAS } \\
\text { 18001:2007 } \\
\text { (BRITISH..., 2007). }\end{array}$} \\
\hline $\begin{array}{l}\text { 2.1. Processo de } \\
\text { implementação do } \\
\text { SGI }\end{array}$ & $\begin{array}{l}\text { As questões buscam identificar quais as etapas realizadas } \\
\text { pelas empresas para a implementação do SGI e investigar } \\
\text { detalhadamente como cada uma destas etapas foi realizada. }\end{array}$ & \\
\hline $\begin{array}{l}\text { 2.2. Integração dos } \\
\text { requisitos das normas }\end{array}$ & $\begin{array}{l}\text { Baseadas na tabela de correspondências entre as normas } \\
\text { ISO 9001 e OHSAS 18001, a qual é apresentada na norma } \\
\text { 18001:2007, as questões buscam identificar como foi } \\
\text { estruturada toda a documentação do sistema de gestão } \\
\text { integrado. As questões devem abordar requisito a requisito } \\
\text { para que possam ser identificados os documentos elaborados } \\
\text { pela organização para o seu atendimento. }\end{array}$ & \\
\hline $\begin{array}{l}\text { 2.3 Estrutura de } \\
\text { gestão do SGI }\end{array}$ & $\begin{array}{l}\text { Questões que visam descrever a estrutura de gestão dos } \\
\text { sistemas de gestão integrados das unidades de análise. }\end{array}$ & \\
\hline
\end{tabular}


Quanto aos documentos verificados, estes se referem a procedimentos, registros e softwares de gestão, todos relacionados ao SGI das empresas. Já os dados provenientes das observações diretas foram obtidos por meio da realização de visitas técnicas.

Baseado nos dados obtidos por meio das três fontes de evidência citadas anteriormente, foi elaborado um relatório com os principais resultados da realização de cada um dos estudos de caso. Estes relatórios foram enviados ao responsável pelo SGI das unidades de análise para validação, o que, segundo Martins (2010), é um mecanismo importante para melhoria da precisão do relatório.

\subsection{Variáveis da pesquisa}

Conforme citado anteriormente, a descrição e a análise da integração dos sistemas de gestão normalizados ISO 9001 e OHSAS 18001 foram realizadas sob três variáveis: processo de implementação do SGI, integração dos requisitos das normas e estrutura de gestão do SGI. O Quadro 2 apresenta os itens verificados durante a execução da pesquisa para cada uma destas variáveis.

A próxima seção apresenta os resultados alcançados com a realização da pesquisa e também a análise dos dados obtidos para cada uma das variáveis de pesquisa apresentadas.

\section{Resultados e análise dos dados}

\subsection{Processo de implementação do SGI}

Quanto ao processo de implementação do sistema de gestão integrado, foi observado que as unidades de análise não seguiram fluxogramas desenvolvidos na literatura para este fim, como o apresentado no trabalho de Karapetrovic (2003). Além disso, no que se refere a uma das etapas do processo de implementação do SGI, a integração dos requisitos das normas, as Unidades de Análise também não utilizaram modelos pré-estabelecidos na literatura, como os apresentados nos trabalhos de Jonker e Karapetrovic (2004) e de Zeng, Shi e Lou (2007). Entretanto, observou-se que as unidades de análise realizaram etapas para a implementação do SGI comuns àquelas apresentadas na bibliografia. Estas etapas são apresentadas no Quadro 3.

Conforme pôde ser observado no quadro, apesar de o número de etapas entre as Unidades de Análise variar, a análise da compatibilidade entre as normas ISO 9001 e OHSAS 18001 é uma etapa comum no processo de implementação do SGI destas empresas. Esta análise pode ser realizada de diversas maneiras, sendo que as Unidades de Análise 1 e 2 realizaram, inicialmente, uma comparação entre os requisitos das normas ISO 9001 e OHSAS 18001 para posterior análise e integração da documentação. A Unidade de Análise 3 utilizou uma tabela de correspondências entre as normas ISO 9001 e OHSAS 18001 para levantar as ações necessárias para a implementação e integração da OHSAS 18001 à ISO 9001. Por fim, a Unidade de Análise 4 utilizou como base para esta análise os processos mapeados da empresa, que foram analisados frente aos requisitos da norma OHSAS 18001 para a sua adequação.

Outro ponto em comum entre as Unidades de Análise foi o passo relacionado à integração da documentação. Para a execução deste passo, as Unidades de Análise utilizaram como base a documentação pré-existente da ISO 9001 para a integração dos requisitos da OHSAS 18001. Na literatura, o modelo apresentado por Karapetrovic (2003), Jonker e Karapetrovic (2004) propõe que os requisitos comuns às normas sejam agrupados em seis processos principais. Já os requisitos específicos, muitos deles únicos à determinada norma, podem ser mantidos separados, formando o que os autores chamam de subsistemas específicos. Os requisitos específicos podem ainda ser agregados aos processos principais estabelecidos.

Entretanto, observando os casos práticos, foi observado que as organizações realizaram a integração de uma maneira simplificada, de forma que o novo

Quadro 2. Variáveis de pesquisa.

\begin{tabular}{|l|l|l|}
\hline \multicolumn{1}{|c|}{ Variável } & \multicolumn{1}{|c|}{ Itens verificados } & \multicolumn{1}{c|}{ Referências } \\
\hline $\begin{array}{l}\text { Processo de } \\
\text { implementação } \\
\text { do SGI }\end{array}$ & $\begin{array}{l}\text { Etapas para a integração do SGI } \\
\text { Detalhamento das etapas para a implementação do SGI }\end{array}$ & $\begin{array}{l}\text { Karapetrovic (2003), Jonker e } \\
\text { Karapetrovic (2004), Zeng, Shi e Lou } \\
\text { (2007) }\end{array}$ \\
\hline $\begin{array}{l}\text { Integração dos } \\
\text { requisitos das } \\
\text { normas }\end{array}$ & $\begin{array}{l}\text { Identificar quais requisitos são integrados e quais } \\
\text { requisitos não são integrados } \\
\text { Identificar as principais características da } \\
\text { documentação do SGI }\end{array}$ & $\begin{array}{l}\text { Salomone (2008), Bernardo et al. } \\
\text { (2009), Jonker e Karapetrovic (2004), } \\
\text { Zeng, Shi e Lou (2007) }\end{array}$ \\
\hline $\begin{array}{l}\text { Estrutura de } \\
\text { gestão do SGI }\end{array}$ & $\begin{array}{l}\text { Verificar a existência de uma estrutura de gestão do SGI } \\
\text { Identificar e entender os papéis e responsabilidades no } \\
\text { gerenciamento do SGI }\end{array}$ & $\begin{array}{l}\text { Donaldson (2007), Bertero (2007), } \\
\text { Lawrence e Lorsch (1973) }\end{array}$ \\
\hline
\end{tabular}


sistema de gestão se adaptasse a uma estrutura de documentação pré-existente. Isto também foi verificado em um trabalho empírico realizado por Salomone (2008), em que os resultados de sua pesquisa mostram que, como as empresas normalmente implementam primeiro a ISO 9001 e depois a ISO 14001 e/ou a OHSAS 18001, a norma que fundamenta a integração dos sistemas de gestão é a ISO 9001.

A realização de treinamentos durante a implementação do SGI também foi uma etapa observada em todas as unidades de análise. Quanto à forma como estes treinamentos aconteceram, observa-se novamente um ponto em comum entre as unidades, dado que em todos os casos foram realizados dois tipos de treinamento: aqueles específicos, referentes aos requisitos exclusivos da OHSAS
18001, e os integrados, referentes aos requisitos comuns entre as normas. Isso porque, apesar de existirem elementos comuns que servem a propósitos parecidos nas normas ISO 9001 e OHSAS 18001, alguns requisitos ainda são peculiares a cada sistema de gestão (PHENG; PONG, 2003).

Por fim, as últimas etapas do processo de integração dos sistemas de gestão referem-se às auditorias interna e externa integradas, as quais podem trazer melhorias tanto para os sistemas de gestão de maneira individual quanto para o sistema de gestão integrado (BERNARDO et al., 2010).

Assim, pode-se dizer que as unidades de análise seguiram basicamente as seguintes etapas para a integração dos sistemas de gestão da qualidade e segurança e saúde ocupacional:

Quadro 3. Etapas para a implementação do sistema de gestão integrado.

\begin{tabular}{|c|c|c|c|c|}
\hline Etapas & $\begin{array}{c}\text { Unidade de } \\
\text { Análise } 1 \\
\end{array}$ & Unidade de Análise 2 & Unidade de Análise 3 & Unidade de Análise 4 \\
\hline 1 & $\begin{array}{c}\text { Análise da } \\
\text { compatibilidade } \\
\text { entre normas ISO } \\
9001 \text { e OHSAS } \\
18001 \text { e integração } \\
\text { da documentação }\end{array}$ & $\begin{array}{c}\text { Planejamento e } \\
\text { comparação entre as } \\
\text { normas }\end{array}$ & $\begin{array}{c}\text { Elaboração do diagnóstico } \\
\text { inicial }\end{array}$ & $\begin{array}{l}\text { Análise da possibilidade } \\
\text { de implementação do SGI } \\
\text { (análise do estado atual, } \\
\text { sem integração, e estado } \\
\text { futuro, normas integradas, } \\
\text { identificação dos } \\
\text { benefícios e dificuldades } \\
\text { decorrentes da integração) }\end{array}$ \\
\hline 2 & $\begin{array}{l}\text { Realização de } \\
\text { treinamentos } \\
\text { (específicos e } \\
\text { integrados) }\end{array}$ & $\begin{array}{c}\text { Análise dos procedimentos } \\
\text { e documentação }\end{array}$ & $\begin{array}{l}\text { Levantamento de } \\
\text { adequações necessárias à } \\
\text { implementação do novo } \\
\text { sistema de gestão }\end{array}$ & $\begin{array}{l}\text { Criação do processo } \\
\text { Sistema de Gestão } \\
\text { Integrado (criação } \\
\text { da estrutura de } \\
\text { gestão, definição de } \\
\text { responsabilidades e } \\
\text { mapeamento integrado dos } \\
\text { processos) }\end{array}$ \\
\hline 3 & $\begin{array}{c}\text { Levantamento } \\
\text { e adequação da } \\
\text { legislação de SSO }\end{array}$ & $\begin{array}{c}\text { Elaboração do cronograma } \\
\text { de integração dos sistemas } \\
\text { de gestão }\end{array}$ & $\begin{array}{l}\text { Elaboração de planos de } \\
\text { ação para realização das } \\
\text { adequações decorrentes } \\
\text { da implementação do SGI } \\
\text { (exemplo: integração da } \\
\text { documentação, realização } \\
\text { de treinamentos) }\end{array}$ & $\begin{array}{l}\text { Realização de treinamentos } \\
\text { (específicos e integrados) }\end{array}$ \\
\hline 4 & $\begin{array}{l}\text { Auditoria Interna } \\
\text { integrada }\end{array}$ & $\begin{array}{l}\text { Integração da OHSAS } \\
18001 \text { ao sistema de gestão } \\
\text { integrado pré-existente de } \\
\text { qualidade e meio ambiente } \\
\text { (adequação e criação de } \\
\text { procedimentos, realização } \\
\text { de treinamentos específicos } \\
\text { e integrados) }\end{array}$ & Auditoria externa integrada & Auditoria externa integrada \\
\hline 5 & $\begin{array}{l}\text { Adequação do } \\
\text { SGI (aspectos } \\
\text { levantados na } \\
\text { auditoria interna) }\end{array}$ & Auditoria interna integrada & & \\
\hline 6 & $\begin{array}{c}\text { Auditoria externa } \\
\text { integrada }\end{array}$ & Auditoria externa integrada & & \\
\hline
\end{tabular}


análise da compatibilidade entre as normas ISO 9001 e OHSAS 18001, análise e integração da documentação, realização de treinamentos e realização das auditorias interna e externa. No fluxograma para a implementação do sistema de gestão integrado, elaborado por Karapetrovic (2003), as etapas sugeridas são: início do processo de implementação, seleção do modelo de integração, seleção de função e normas para a integração, integração dos requisitos das normas, realização de análise de lacunas (que inclui o alinhamento e integração da documentação dos objetivos e dos processos e recursos) e melhoria do sistema de gestão integrado.

Observa-se que, nas Unidades de Análise que compõem este estudo, todas estas etapas foram seguidas, exceto a escolha de um modelo de integração, dado que as empresas não conheciam tais modelos. Assim, todas as unidades de análise selecionaram as normas a serem integradas, observaram a integração dos requisitos das normas por meio da tabela de correspondências (BRITISH..., 2007) ou por meio da análise de seus processos, realizaram a análise de lacunas e, por meio da realização de auditorias, identificaram melhorias.

Apresentados os resultados e a análise da primeira variável de pesquisa, processo de implementação do SGI, o próximo tópico apresenta os resultados relacionados à segunda variável, integração dos requisitos das normas.

\subsection{Integração dos requisitos das normas}

Para a análise de como ocorre a integração dos requisitos das normas ISO 9001 e OHSAS 18001, as unidades de análise foram questionadas em relação à documentação do sistema de gestão integrado. Isto porque, usualmente, a maneira como a organização atende aos requisitos de determinada norma de sistema de gestão pode ser verificada por meio de procedimentos escritos.

Assim, por meio da análise da documentação, observou-se que o Sistema de Gestão Integrado das Unidades de Análise é composto basicamente por cinco tipos de documentos, com características específicas. No primeiro tipo, a descrição do atendimento aos requisitos da ISO 9001 e da OHSAS 18001 é realizada em um único documento, o qual não faz referência a procedimentos ou instruções técnicas específicas relacionados à uma determinada norma. Geralmente, este tipo de documento trata de requisitos comuns entre os sistemas de gestão, como o controle de documentos, controle de registros e auditoria interna.

$\mathrm{O}$ segundo tipo de documento refere-se àqueles requisitos específicos da área de qualidade, mas que possuem correspondência com vários requisitos de SSO. Assim, estes documentos apresentam questões relacionadas à OHSAS 18001, sem grande detalhamento, e referenciam procedimentos/instruções técnicas específicos desta norma. Os requisitos de projeto e desenvolvimento da ISO 9001 ilustram bem este tipo de documentação, dado que o procedimento para seu atendimento, além de considerar o requisito de controle operacional da OHSAS (4.4.6), ainda deve considerar um requisito específico desta norma, o 4.3.1, que se refere à identificação de perigos, avaliação de riscos e determinação de controles.

O terceiro tipo de documento refere-se exclusivamente aos requisitos específicos das normas ISO 9001 e OHSAS 18001, nos quais são tratados assuntos referentes apenas ao atendimento de requisitos de determinada norma. Estes documentos geralmente são aqueles referenciados em documentos do segundo tipo, os quais foram descritos anteriormente. Por fim, o quarto e o quinto tipo de documentos referem-se, respectivamente, aos registros integrados e específicos. Os registros integrados demonstram que, para a realização de uma determinada atividade, tanto os requisitos da ISO 9001 quanto da OHSAS 18001 foram observados. Já os registros específicos relacionam-se apenas à realização de atividades que envolvem requisitos de uma determinada norma.

O Quadro 4 apresenta quais requisitos as unidades de análise integram. Para definir quais requisitos são integrados e quais requisitos não são integrados, foram utilizados como referência apenas os três primeiros tipos de documentos identificados. Assim, se a documentação principal é do primeiro ou do segundo tipo, considerou-se que os requisitos são integrados e, se a documentação principal é do terceiro tipo, considerou-se que os requisitos não são integrados. Na ausência de procedimentos escritos, as organizações foram questionadas sobre a prática utilizada para o atendimento dos requisitos das normas.

Conforme citado na revisão bibliográfica deste trabalho, a integração dos requisitos dos sistemas de gestão é discutida nos trabalhos de Salomone (2008) e Bernardo et al. (2009). No trabalho de Salomone (2008) os requisitos citados como os mais integrados pelas organizações são: controle de documentos, política, análise crítica, objetivos e metas, controle de registros, auditoria interna e manual. Observando o Quadro 4, é possível verificar que, nas Unidades de Análise, esta integração também acontece, exceto pelo requisito relacionado à definição de objetivos, pois conforme declarado pelos entrevistados, apesar de os objetivos partirem de uma política integrada estes estão claramente definidos para cada um dos sistemas de gestão de maneira individual, não existindo objetivos integrados.

Analisando o Quadro 4 em relação aos níveis de integração propostos por Sampaio, Saraiva e Domingues (2012), que seriam integração da documentação (nível 1), integração das ferramentas de gerenciamento (nível 2), políticas e objetivos comuns 
Quadro 4. Integração dos requisitos das normas ISO 9001 e OHSAS 18001.

\begin{tabular}{|c|c|c|c|c|}
\hline \multirow[t]{2}{*}{ Requisitos } & \multicolumn{4}{|c|}{$\begin{array}{l}\text { Unidades de } \\
\text { análise }\end{array}$} \\
\hline & 1 & 2 & 3 & 4 \\
\hline Controle de documentos e registros (ISO 4.2.3, 4.2.4 e OHSAS 4.4.5, 4.5.4) & $\mathrm{X}$ & $\mathrm{X}$ & $\mathrm{X}$ & $\mathrm{X}$ \\
\hline Política (ISO 5.3 e OHSAS 4.2) & $\mathrm{X}$ & $\mathrm{X}$ & $\mathrm{X}$ & $\mathrm{X}$ \\
\hline \multicolumn{5}{|l|}{ Objetivos (ISO 5.4.1 e OHSAS 4.3.3) } \\
\hline Comunicação (ISO 5.5.3 e OHSAS 4.4.3) & & $\mathrm{X}$ & $\mathrm{X}$ & $\mathrm{X}$ \\
\hline Provisão de recursos (ISO 6, 6.1 e OHSAS 4.4.1) & & $\mathrm{X}$ & & \\
\hline Realização do produto (ISO 7) e implementação e operação (OHSAS 4.4) & & $\mathrm{X}$ & $\mathrm{X}$ & \\
\hline Planejamento da realização do produto (ISO 7.1) e controle operacional (OHSAS 4.4.6) & & $\mathrm{X}$ & $\mathrm{X}$ & $\mathrm{X}$ \\
\hline $\begin{array}{l}\text { Processos relacionados a clientes e determinação e análise crítica dos requisitos relacionados } \\
\text { ao produto (ISO 7.2, 7.2.1, 7.2.2), controle operacional (OHSAS 4.4.6), Identificação de } \\
\text { perigos, avaliação de riscos e determinação de controles (OHSAS 4.3.1) e requisitos legais e } \\
\text { outros (OHSAS 4.3.2) }\end{array}$ & $\mathrm{X}$ & $\mathrm{X}$ & & $\mathrm{X}$ \\
\hline $\begin{array}{l}\text { Projeto e desenvolvimento (ISO 7.3, 7.3.1, 7.3.2, 7.3.3, 7.3.4, 7.3.5, 7.3.6, 7.3.7) e controle } \\
\text { operacional (OHSAS 4.4.6) }\end{array}$ & $\mathrm{X}$ & $\mathrm{X}$ & $\mathrm{X}$ & $\mathrm{X}$ \\
\hline Aquisição (ISO 7.4, 7.4.1, 7.4.2 e 7.4.3) e controle operacional (OHSAS 4.4.6) & $\mathrm{X}$ & $\mathrm{X}$ & $\mathrm{X}$ & $\mathrm{X}$ \\
\hline Produção e prestação de serviço (ISO 7.5, 7.5.1, 7.5.2) e controle operacional (OHSAS 4.4.6) & $\mathrm{X}$ & NA & NA & $\mathrm{X}$ \\
\hline $\begin{array}{l}\text { Preservação do produto manuseio, conservação e embalagem (ISO 7.5.5) e controle } \\
\text { operacional (OHSAS 4.4.6) }\end{array}$ & $\mathrm{X}$ & $\mathrm{X}$ & $\mathrm{X}$ & $\mathrm{X}$ \\
\hline Auditoria interna (ISO 8.2.2 e OHSAS 4.5.5) & $\mathrm{X}$ & $\mathrm{X}$ & $\mathrm{X}$ & $\mathrm{X}$ \\
\hline Não conformidades e ações corretivas/preventivas (ISO 8.3, 8.5.2, 8.5.3 e OHSAS 4.5.3.2, 4.4.7) & $\mathrm{X}$ & $\mathrm{X}$ & $\mathrm{X}$ & \\
\hline Análise crítica da direção (ISO 5.6, 5.6.1, 5.6.2, 5.6.3 e OHSAS 4.6) & $\mathrm{X}$ & $\mathrm{X}$ & $\mathrm{X}$ & $\mathrm{X}$ \\
\hline
\end{tabular}

(nível 3) e estrutura organizacional comum (nível 4), observa-se que nas organizações pesquisadas não se pode afirmar de maneira exata em qual desses níveis cada empresa se encontra. Apesar de todas as empresas elas integrarem grande parte de sua documentação (nível 1) e ferramentas de gestão (nível 2), como a análise crítica da direção, os requisitos para se alcançar o nível 3 não são totalmente atendidos. Isso porque as políticas são integradas, mas os objetivos não são integrados em nenhuma das unidades de análise. Já a questão da estrutura organizacional varia de acordo com o porte da organização, o que será detalhado posteriormente (no item 4.3 Estrutura de gestão do SGI). Assim, verifica-se que existe uma dificuldade em identificar o nível de integração em que estas empresas se encontram, mostrando que a transição de um nível para outro pode não se dar de maneira completa.

Bernardo et al. (2009) buscaram identificar o grau de integração entre os requisitos das normas (não integrado, parcialmente integrado e totalmente integrado), sendo que aqueles requisitos apontados pela maioria das empresas como totalmente integrados foram: controle de documentos e registros, análise crítica, auditoria interna, comunicação interna, controle de não conformidades e ação corretiva/preventiva. Os requisitos de realização do produto foram apontados como tendo o menor grau de integração. Neste trabalho, os requisitos com maior grau de integração, citados por Bernardo et al. (2009), são integrados na maioria das Unidades de Análise e os requisitos relacionados à realização do produto, citados como tendo um baixo grau de integração, são integrados nas Unidades de Análise 2 e 3.

Apesar de não terem sido apresentados no Quadro 4, é necessário observar que os requisitos específicos da OHSAS 18001 (como preparação e atendimento de emergências e investigação de acidentes) e da ISO 9001 (como identificação e rastreabilidade e satisfação do cliente) não são integrados e referem-se à procedimentos específicos que tratam apenas do atendimento a estes requisitos.

Analisando as informações referentes aos tipos de documentos identificados e à integração dos requisitos, pode-se dizer que as Unidades de Análise, assim como proposto pelo modelo para a integração de sistemas de gestão baseado na abordagem de sistemas, desenvolvido por Karapetrovic (2003), Jonker e Karapetrovic (2004), formaram dentro de seu sistema de gestão integrado, subsistemas específicos, os quais relacionam-se às particularidades de cada norma. Considera-se que a formação desses subsistemas seja um aspecto comum dos sistemas de gestão integrados, dado que, apesar da compatibilidade existente entre a ISO 9001 e a OHSAS 18001, alguns requisitos ainda são peculiares aos objetivos de cada norma (PHENG; PONG, 2003; ZENG; SHI; LOU, 2007), como é o caso dos requisitos de identificação 
de perigos, avaliação de riscos e determinação de controles da OHSAS 18001 e o requisito de satisfação do cliente da ISO 9001.

Observou-se também que a integração dos requisitos das normas e como ela ocorre pode depender de diversos fatores, como os modelos pré-estabelecidos pela organização, padrões utilizados, quantidade de regras e documentação, dentre outros (DONALDSON, 2007). A existência desses modelos faz com que as organizações não integrem, por exemplo, alguns formulários, mesmo que o procedimento a que se refere este formulário seja integrado. Como exemplo, pode-se citar a Unidade de Análise 2, que, apesar de possuir um procedimento de controle de não conformidades integrado, possui formulários para registro das não conformidades de qualidade e de SSO distintos. Isso porque o formulário para o registro de não conformidades de SSO segue um padrão corporativo.

$\mathrm{Na}$ Unidade de Análise 1, os formulários para registro de não conformidades também não são integrados, sendo que, por meio deste exemplo, é possível visualizar mais uma vez a interferência dos padrões utilizados pela organização na integração. Questionado sobre a possibilidade de integração dos formulários para registro de não conformidade, o entrevistado ressaltou que esta integração não seria interessante. Isso porque o formulário para o registro de não conformidades de qualidade possui várias vias, informações técnicas sobre a não conformidade, entre outros campos considerados importantes pelo controle de qualidade. Já o formulário para registro de não conformidades de SSO foi formulado para ser simples e de fácil preenchimento por qualquer funcionário.

Segundo Zutshi e Sohal (2005), considerar as similaridades entre as normas para a integração é importante, mas reconhecer as diferenças é igualmente consequencial. Analisando a forma como a integração dos requisitos das normas foi realizada nas Unidades de Análise, observa-se que, tanto as similaridades quanto as diferenças entre as normas ISO $9001 \mathrm{e}$ OHSAS 18001 foram consideradas, o que resultou na elaboração de documentos integrados e específicos para cada sistema de gestão.

Apresentada a análise dos resultados referentes à variável sobre a integração dos requisitos das normas nas Unidades de Análise, a próxima subseção apresenta outro aspecto importante para a implementação de um SGI, a estrutura desenvolvida pelas organizações para o seu gerenciamento.

\subsection{Estrutura de gestão do SGI}

As Unidades de Análise 1, 2, 3 e 4 estabeleceram diferentes estruturas para o gerenciamento e operacionalização das atividades do sistema de gestão integrado. Isso porque, a forma como acontece a coordenação entre os departamentos de uma organização pode variar, pois isto depende de fatores contingenciais não só relacionados ao ambiente industrial (LAWRENCE; LORSCH, 1973), mas também de questões como o processo de tomada de decisões, o estabelecimento de padrões para comunicação ou comportamento e o tamanho da organização (DONALDSON, 2007; BERTERO, 2007). Em relação ao tamanho da organização, observou-se uma variação da estrutura de gestão entre as Unidades de Análise, uma vez que quanto maior o número de funcionários mais complexa e formal a estrutura de gestão. É importante ressaltar que esta diferença já era esperada, pois, conforme discutido por vários autores (BLAU; SCHOENHERR, 1971; CHILD; MANSFIELD, 1972; MINTZBERG, 1989, p. 106; PUGH et al., 1968) apud (MORENO-LUZON; PERIS, 1998) diferentes tamanhos de organização significam diferentes graus de dificuldade no planejamento, controle e coordenação das atividades internas da empresa. Assim, empresas maiores estão relacionadas a um número maior de regras e procedimentos e a formas mais exaustivas de planejamento e controle.

Para a realização desta análise, é necessário destacar que as unidades de Análise 1, 2, 3 e 4 contam, respectivamente, com aproximadamente 800, 400, 1.570 e 7.700 funcionários. Assim, iniciando-se pela Unidade de Análise 2, que possui o menor número de funcionários (400), observa-se que a estrutura de gestão é formada por dois profissionais, um da área de qualidade (supervisor de gestão da qualidade) e um da área de segurança e saúde ocupacional (engenheiro de segurança). Estes funcionários são responsáveis pelo gerenciamento do sistema de gestão integrado, sendo que o supervisor de gestão da qualidade é responsável pelo gerenciamento e pela implementação dos requisitos comuns entre a ISO 9001 e a OHSAS 18001 e pela implementação dos requisitos específicos da ISO 9001.

Já o engenheiro de segurança é responsável pelo gerenciamento e execução de atividades operacionais relacionadas apenas à OHSAS 18001. É importante ressaltar ainda, que ambos os funcionários são Representantes da Direção (RD) na organização, sendo o supervisor de gestão da qualidade o RD para a ISO 9001 e o engenheiro de segurança o RD para a OHSAS 18001.

$\mathrm{Na}$ Unidade de Análise 1, empresa com 800 funcionários, observa-se uma pequena alteração na estrutura de gestão. As atividades de gerenciamento do sistema de gestão integrado são centralizadas no gerente da qualidade/lean production, reconhecido na organização como o gestor do sistema de gestão integrado. A operacionalização dos requisitos comuns entre as normas também é de responsabilidade deste gestor, já o atendimento aos requisitos operacionais 
específicos da OHSAS 18001 são de responsabilidade do pessoal da área de segurança e saúde ocupacional.

Na Unidade de Análise 3 (1.570 funcionários), a estrutura de gestão envolve uma área específica, um gerente e três grupos distintos de funcionários, entre os quais as atividades do sistema de gestão integrado são dividas. Esta área específica é chamada de Competitividade, e é responsável, entre outras atividades, pelo gerenciamento das normas e das certificações que a organização possui. Esta área está ligada a um gerente executivo, que possui, entre suas responsabilidades, a manutenção das certificações. Os três grupos formados na organização para a execução das atividades de manutenção do sistema de gestão integrado são os comitês, subcomitês e os grupos de trabalho.

Os comitês são responsáveis por questões consideradas estratégicas, como a implementação de novas normas na organização. Os subcomitês são responsáveis pela realização das reuniões de análise crítica e pela análise e acompanhamento da implementação dos requisitos das normas, atividades consideradas de caráter gerencial. Por fim, os grupos de trabalho são responsáveis por acompanhar a operacionalização da implementação dos sistemas de gestão, o que envolve atividades como o acompanhamento do tratamento de não conformidades.

Na Unidade de Análise 4, empresa com o maior número de funcionários (7.700), a estrutura de gestão conta com um Diretor do Sistema de Gestão Integrado e dois coordenadores que respondem a este diretor. Quanto aos coordenadores, um é responsável pela gestão da qualidade e outro pela gestão da segurança e saúde ocupacional. Suas responsabilidades convergem em relação à implementação e gerenciamento dos requisitos comuns entre as normas ISO 9001 e OHSAS 18001, já a implementação e gerenciamento dos requisitos específicos de cada norma são divididos entre esses coordenadores.

Assim, observando-se a estrutura de gestão das Unidades de Análise que compõem este estudo e tomando-se como variável contingencial o tamanho da organização, é possível verificar que quanto maior o número de funcionários, mais complexa a estrutura de gestão. A esta estrutura de gestão, que no caso deste trabalho visa coordenar as áreas do Sistema de Gestão Integrado, Lawrence e Lorsch (1973) dão o nome de unidades integradoras. Segundo os autores, as unidades integradoras em uma organização podem ser mais ou menos formais. Em relação a este aspecto, neste estudo, observou-se também que quanto maior o número de funcionários mais formal é a estrutura de gestão. Na Unidade 2, a responsabilidade pelo gerenciamento do SGI é do supervisor de gestão da qualidade e do engenheiro de segurança, já na Unidade de Análise 1, esta responsabilidade passa a um gerente. Na Unidade de Análise 3, há uma área e um gerente executivo responsáveis pelo SGI e, por fim, na Unidade de Análise 4, há um diretor do sistema de gestão integrado e dois coordenadores responsáveis pela sua gestão.

Realizada a análise da integração dos sistemas de gestão normalizados ISO 9001 e OHSAS 18001 sob as três variáveis de pesquisa propostas, a próxima seção apresenta as principais conclusões deste trabalho.

\section{Conclusões}

O objetivo deste trabalho foi descrever e analisar a integração dos sistemas de gestão normalizados ISO 9001 e OHSAS 18001. Esta análise foi realizada sob três variáveis: quanto ao processo de implementação do SGI, quanto à integração dos requisitos das normas e quanto à estrutura de gestão do SGI.

Observando os resultados obtidos, pode-se dizer que o processo de integração dos sistemas de gestão ISO 9001 e OHSAS 18001 nas empresas estudadas apresenta diversas similaridades. Exemplo disso, são os passos realizados para a implementação do SGI e as características da documentação desenvolvida pelas empresas para atender aos requisitos das normas. Apesar das similaridades entre os documentos desenvolvidos, a forma como estes estão estruturados está relacionada a padrões organizacionais, os quais definem não apenas o formato dos procedimentos, instruções técnicas e registros, mas também a sua integração.

Observou-se ainda que a estrutura de gestão desenvolvida em cada organização adapta-se à variável "número de funcionários", a qual influencia na sua complexidade e formalidade. A integração dos sistemas de gestão pressupõe a coordenação de esforços entre diferentes áreas, tanto para a implementação de requisitos comuns às normas, quanto para o gerenciamento do SGI. Se esta coordenação não ocorre, ou ocorre de maneira inadequada, corre-se o risco da realização de atividades redundantes, ocasionando conflitos entre as áreas que compõem o SGI.

Quanto às contribuições desta pesquisa, estas foram identificadas tanto para a área acadêmica quanto para a área gerencial. Para a área acadêmica, foram identificadas contribuições referentes a cada uma das três variáveis de pesquisa, utilizadas para a realização da análise da integração dos sistemas de gestão normalizados ISO 9001 e a OHSAS 18001. Quanto ao processo de implementação do SGI (primeira variável) foi possível observar como este acontece na prática, dado que a bibliografia apresenta apenas modelos conceituais de implementação. Outra contribuição refere-se à verificação de que as empresas não utilizam modelos conceituais para a integração dos sistemas de gestão normalizados. A bibliografia apresenta diversos modelos para este fim, entretanto, observou-se que as empresas realizam esta integração 
com base na documentação pré-existente de sistemas de gestão normalizados já implementados, facilitando assim este processo.

Quanto à integração dos requisitos das normas (segunda variável), identificou-se como esta ocorre por meio da análise da documentação desenvolvida pelas empresas para a implementação dos requisitos do SGI. Em outros trabalhos, são apresentados apenas quais requisitos são ou não integrados e em que grau. Este trabalho, além de mostrar quais requisitos são integrados, contribui no sentido de apresentar de maneira detalhada como esta integração ocorre, o que foi realizado por meio da verificação da documentação das empresas. A partir deste detalhamento, foi possível observar similaridades do SGI das organizações com modelos para a integração de requisitos apresentados na bibliografia, como a formação de subsistemas específicos no SGI relacionados a cada norma que o compõe. Foi possível observar ainda, conforme apresentado em trabalhos teóricos, que padrões corporativos, como modelos pré-estabelecidos de determinados documentos, influenciam na integração da documentação.

Em relação à estrutura de gestão do SGI (terceira variável), observou-se a influência da variável contingencializadora, tamanho da organização, na complexidade e na formalidade desta estrutura. A bibliografia apenas cita a importância da existência de uma estrutura de gestão do SGI, mas não mostra como isso acontece nas organizações. Assim, este trabalho contribui não apenas mostrando como as empresas gerenciam o SGI, mas também identificando a existência da influência de variáveis contingencializadas em sua complexidade e formalidade.

Outra contribuição deste trabalho é a possibilidade da análise da questão da integração a partir dos dados empíricos obtidos, uma vez que os artigos já publicados na literatura da área não apresentam esse tipo de dado, mas tratam de questões como metodologias para a implementação de sistemas de gestão integrados, buscam identificar benefícios e desvantagens do SGI, analisar a compatibilidade entre as normas de sistemas de gestão ou apenas identificar se as empresas integram ou não os requisitos de seus sistemas de gestão. É importante salientar que os trabalhos sobre a integração dos requisitos dos sistemas de gestão se limitam apenas à verificação da ocorrência ou não desta integração, não apresentando dados detalhados sobre o processo.

Por meio dos dados empíricos obtidos com este trabalho, é possível questionar, por exemplo, até que ponto a integração é desejável, uma vez que foram observadas desvantagens com a implementação do sistema de gestão integrado. Uma destas desvantagens refere-se à realização das auditorias de maneira integrada, as quais são consideradas superficiais por alguns entrevistados. Acredita-se que as empresas devem realizar uma análise cuidadosa antes de integrar as auditorias internas e externas, pois, apesar da redução do custo e do tempo para a sua realização, pode-se não obter os mesmos resultados de uma auditoria que é realizada com o foco em apenas um sistema de gestão. Segundo Heras-Saizarbitoria e Boiral (2012), a realização de auditorias por terceiros deve receber mais atenção dos gestores que devem acompanhá-las e analisá-las quanto á sua qualidade, prevenindo assim prejuízos à organização. Quanto às limitações da pesquisa, destaca-se a utilização do método de estudo de caso, o qual impossibilita a generalização dos resultados, uma vez que, a realização do estudo de caso requer um tempo considerável para a sua realização, o que impede que uma amostra com muitas unidades de análise seja utilizada. Quanto aos trabalhos futuros, sugere-se que o escopo deste trabalho seja ampliado, ou seja, que a investigação de como acontece a integração dos sistemas de gestão normalizados envolva outros sistemas de gestão implementados pelas organizações, não apenas a ISO 9001 e a OHSAS 18001.

\section{Referências}

ASSOCIAÇÃO BRASILEIRA DE NORMAS TÉCNICAS - ABNT. Normalização. Disponível em: <http://www.abnt.org.br>. Acesso em: dez. 2009.

ASSOCIAÇÃO BRASILEIRA DE NORMAS TÉCNICAS - ABNT. Sistemas de Gestão da Qualidade: Requisitos. 2. ed. Rio de Janeiro: ABNT, 2008. 28 p.

BERNARDO, M. et al. Do integration difficulties influence management system integration levels? Journal of Cleaner Production, v. 21, n. p. 23-33, 2012.

BERNARDO, M. et al. How integrated are environmental, quality and other standardized management systems? an empirical study. Journal of Cleaner Production, v. 17, n. 8, p. 742-750, 2009. http://dx.doi.org/10.1016/j. jclepro.2008.11.003

BERNARDO, M. et al. An empirical estudy on the integration of management system audits. Journal of Cleaner Production, v. 18, n. 5, p. 486-495, 2010. http://dx.doi.org/10.1016/j.jclepro.2009.12.001

BERTERO, C. O. Nota técnica: Teoria da contingência estrutural. SãoPaulo: Atlas, 2007. p. 132-134.

BRITISH RETAIL CONSORTIUM. 2010. Disponível em: $<$ http://www.brc.org.uk/>.

BRITISH STANDARDS INSTITUTION. Occupational Health and Safety Management Systems: requirements. OHSAS Project Group-British Standards Institution, 2007. $34 \mathrm{p}$.

CHURCHMAN, C. W. Introdução à Teoria dos Sistemas. 2. ed. Editora Vozes, 1972. 309 p.

DESSLER, G. Organization and Management: A Contingency Approach. London: Prentice-Hall, 1976.

DONALDSON, L. Teoria da contingência estrutural. São Paulo: Atlas, 2007. p. 105-131.

FRESNER, J.; ENGELHARDT, G. Experiences with integrated management systems for two small companies 
in austria. Journal of Cleaner Production, v. 12, n. 6, p. 623-631, 2004. http://dx.doi.org/10.1016/j. jclepro.2003.09.013

HERAS-SAIZARBITORIA, I.; BOIRAL, O. ISO 9001 and ISO 14001: Towards a Research Agenda on Management System Standards. International Journal of Management Reviews, 2012. Early View (Online Version of Record published before inclusion in an issue). http://dx.doi.org/10.1111/j.1468-2370.2012.00334.x

INTERNATIONAL ORGANIZATION FOR STANDARDIZATION. Sítio da International Organization for Standardization. 2009. Disponível em: <http://www.iso.org/>.

INTERNATIONAL ORGANIZATION FOR STANDARDIZATION. ISO Concept Database. 2010. Disponível em: http://cdb.iso.org.

JONKER, J.; KARAPETROVIC, S. Systems thinking for the integration of management systems. Business Process Management Journal, v. 10, n. 6, p. 608-615, 2004. http://dx.doi.org/10.1108/14637150410567839

KARAPETROVIC, S. Musings on integrated management systems. Measuring Business Excelence, v. 7, n. 1, p. 4-13, 2003. http://dx.doi. org/10.1108/13683040310466681

KARAPETROVIC, S.; WILLBORN, W. Integration of quality and environmental management systems. The TQM Magazine, v. 10, n. 3, p. 204-213, 1998. http:// dx.doi.org/10.1108/09544789810214800

KAUSEK, J. OHSAS 18001 Designing and Implementing an Effective Health and Safety Management System. Lanham: The Rowmanand Little?eld Publishing Group, 2007. 162 p.

LAWRENCE, P. R.; LORSCH, J. W. As empresas e o ambiente: Diferenciação e Integração Administrativas. Petrópolis: Vozes, 1973. 300 p.

MARTINS, R. A. Abordagens quantitativa e qualitativa. Rio de Janeiro: Elsevier, 2010. p. 45-61.

MIGUEL, P. A. C. Estudo de caso na engenharia de produção: estruturação e recomendações par a sua condução. Produção, v. 17, p. 216-229, 2007. http:// dx.doi.org/10.1590/S0103-65132007000100015

MIGUEL, P. A. C. Adoção do estudo de caso na engenharia de produção. Rio de Janeiro: Elsevier, 2010. p. 129-143.

MORENO-LUZÓN, M. D.; PERIS, F. J. Strategic approaches, organizational design: integration in a fit and contingency model. International Journal of Quality Science, v. 3, n. 4, p. 328-347, 1998. http:// dx.doi.org/10.1108/13598539810243667

RIBEIRO NETO, J. B. M.; TAVARES, J. C.; HOFFMANN, S. C. Sistemas de Gestão Integrados: qualidade, meio ambiente, responsabilidade social e segurança e saúde no trabalho. São Paulo: Senac, 2008. 324 p.

ØRGENSEN, T. H. J.; REMMEN, A.; MELLADO, M. D. Integrated management systems - three different levels of integration. Journal of Cleaner Production, v. 14, n. 8, p. 713-722, 2006. http://dx.doi.org/10.1016/j. jclepro.2005.04.005

PHENG, L. S.; KWANG, G. K. Iso 9001, ISO 14001 and OHSAS 18001 management systems: Integration, costs and bene?ts for construction companies. Architectural Science Review, v. 48, p. 145-152, 2005. http://dx.doi. org/10.3763/asre.2005.4818

PHENG, L. S.; PONG, C. Y. Integrating iso 9001 and ohsas 18001 for construction. Journal of Construction Engineering and Management, v. 129, n. 3, p. 338-347, 2003. http://dx.doi.org/10.1061/ (ASCE)0733-9364(2003)129:3(338)

POJASEK, R. B. Is your integrated management system really integrated? Environmental Quality Management, v. 16, n. 2, p. 89-97, 2006. http://dx.doi.org/10.1002/ tqem. 20124

POUPART, J. et al. A pesquisa qualititiva: Enfoques epistemológicos e metodógicos. Petrópolis: Vozes, 2008.

SALOMONE, R. Integrated management systems - experiences in italian organizations. Journal of Cleaner Production, v. 16, n. 6, p. 1786-1806, 2008. http://dx.doi.org/10.1016/j.jclepro.2007.12.003

SAMPAIO, P.; SARAIVA, P.; DOMINGUES, P. Management systems: integration or addition? International Journal of Quality \& Reliability Management, v. 29, n. 4, p. 402-424, 2012. http:// dx.doi.org/10.1108/02656711211224857

TRICKER, R. ISO 9001:2000 for Small Businesses. 3. ed. Oxford: Elsevier Butterworth Heinemann, 2007. 520 p.

WILKINSON, G.; DALE, B. Integrated management systems - an examination of the concept and theory. The TQM Magazine, v. 11, n. 2, p. 95-104, 1999. http:// dx.doi.org/10.1108/09544789910257280

YIN, R. K. Estudo de Caso: Planejamento e Métodos. 3. ed. Bookman, 2005. 212 p.

ZENG, S. X.; SHI, J. J.; LOU, G. X. A synergetic model for implementing an integrated management system-an empirical study in China. Journal of Cleaner Production, v. 15, n. 18, p. 1760-1767, 2007. http:// dx.doi.org/10.1016/j.jclepro.2006.03.007

ZUTSHI, A.; SOHAL, A. S. Integrated management system - the experiences of three australian organisations. Journal of Manufacturing Technology Management, v. 16, n. 2, p. 211-232, 2005. 